$\xi=-1$

\title{
Spatial Three Degree of Freedom Parallel Manipulator Forward Kinematic Position Analysis
}

\author{
Srinivasa Rao Pundru ${ }^{1 *}$, Mohan Rao Nalluri ${ }^{2}$ \\ ${ }^{1}$ Srinivasa Rao Pundru, Associate Professor, Department of Mechanical Engineering, \\ Mahatma Gandhi Institute of Technology, C.B.I.T(post), Gandipet, Hyderabad-500 075, Telangana State, India \\ ${ }^{2}$ Mohan Rao Nalluri, Professor, Department of Mechanical Engineering, JNTUK, Kakinada-533 003, A.P., India \\ ${ }^{*}$ Corresponding author E-mail: srinupundru@gmail.com
}

\begin{abstract}
This work presents forward kinematic position analysis of a spatial three degree of freedom parallel manipulator, which has three symmetric loops. The three loops consist of an actuated sliding links- rotational and spherical joints. The actuated sliding links are attached to inclined base platform via rotational joints. The limbs are connected from rotational joints to moving platform by spherical joints. The degree of freedom of a spatial parallel manipulator is analyzed via kutzbach criterion. The forward kinematic position analysis carried out by using 3-coupled trigonometric equations which are formulated with side and behaviour constraints of the manipulator. There are many difficulties in solving the system of non-linear equations in kinematics of manipulator therefore by using MATLAB the three nonlinear coupled algebraic equations are solved. The forward position kinematic analysis part is used in the development procedure of spatial parallel manipulator to check, the required and obtained positions of the moving platform of the developed manipulator.
\end{abstract}

Keywords: Forward kinematics; position analysis; spatial parallel manipulator; 3- degree of freedom.

\section{Introduction}

The parallel manipulators perform the task of controlling the moving platform with respect to the base frame. The six degree of freedom spatial parallel manipulators such as Stewart -Gough platform type and Stewart universal type test machines [1,2] suffer the difficult in position kinematic analysis. For several applications like air craft simulation require less than 6 -dof. Therefore, less than six degree of freedom spatial parallel manipulators have been developed. Yangmin $\mathrm{Li}$ and QingsongXu[3,4] proposed kinematic analysis of 3-dof translational parallel manipulators which are moving in rectangular coordinate system. Meng-Shiun T sai[5] proposed the analysis of a 3-PRS parallel mechanism which indicate the analysis of mechanism. J.A.carretero [6] proposed analysis and optimization of parallel manipulator in which prismatic joints are actuated. Lee and Shah[7] proposed analysis of 3-dof parallel actuated manipulator, in which the motion will be constrained .Yangmin $\mathrm{Li}$ and QingsongXu[8] presented the analysis of 3-PRS parallel manipulator in which the sliding joints were actuated. Xin-JunLiu and Farhad Tahmasebi[9,10] performed new parallel manipulators using the Grubler mobility criterion as it may be demonstrated that the mechanism has 3-dof. The purpose of this work is to develop an analytical method and systematic design procedure to analyze the basic forward position kinematics of 3-dof spatial parallel manipulator. The geometric method is applied to formulate 3-coupled trigonometric equations by considering with side and behaviour constraints and equations are solved by vector technique. The nonlinear equations which are very difficult to solve, therefore by using MATLAB the three non-linear coupled algebraic kinematic equations are solved.

\section{Geometry Description of Spatial 3-Dof Par- allel Manipulator}

The spatial three degree of freedom parallel manipulator consists of a moving platform which is connected to a fixed base by three similar supporting limbs with three symmetric loops as shown in Fig 1. In these three loops the number of rotational joints, type of joints and number of sliding joints and number of moving limbs are same and equal to the dof of the moving platform of the manipulator. The actuated sliding joint of each limb is inclined from the fixed link of base platform by an angle $\alpha_{\mathrm{ji}}$ for $i^{\text {th }}$ position of limb. The sliding joint is actuated on a limb of fixed length via a rotational joint and limbs are connected to the moving platform by spherical joints. The fixed platform co-ordinate reference frame $\mathrm{O}\{\mathrm{x}, \mathrm{y}, \mathrm{z}\}$ is considered at the centre of $\Delta^{\mathrm{le}} \mathrm{B}_{1} \mathrm{~B}_{2} \mathrm{~B}_{3}$. Similarly the moving platform co-ordinate reference frame $\mathrm{P}\{\mathrm{u}, \mathrm{v}, \mathrm{w}\}$ is considered at the centre of $\Delta^{\mathrm{le}} \mathrm{S}_{1} \mathrm{~S}_{2} \mathrm{~S}_{3}$. Consider $\mathrm{x}$-axis in the direction $\overrightarrow{\mathrm{OB}_{1}}$ and the $\mathrm{u}$-axis in the direction $\overrightarrow{\mathrm{PS}_{1}}$. For each actuation along three loops the distance between $B_{j}$ and $P_{R_{j}}$ is denoted by $\left.\overrightarrow{d_{1}}\right|_{j=1,2,3 \text { and } i=1,2,3,4 \text { etc }}$ as in Fig. 1. The Fig.2 and Fig. 3 represent the position vector of fixed platform vertices $\left.B_{j}\right|_{j=1,2,3}$ and moving platform vertices $\left.S_{j}\right|_{j=1,2,3}$ with respect to fixed base frame centre $\mathrm{O}\{\mathrm{x}, \mathrm{y}, \mathrm{z}\}$ and moving platform frame centre $\mathrm{P}\{\mathrm{u}, \mathrm{v}$, w $\}$ can be expressed as follows:

$\mathrm{OB}_{1}=\mathrm{g}_{1}, \mathrm{OB}_{2}=\mathrm{g}_{2}, \mathrm{OB}_{3}=\mathrm{g}_{3}, \mathrm{OS}_{1}=\mathrm{h}_{1}, \mathrm{OS}_{2}=\mathrm{h}_{2}, \mathrm{OS}_{3}=$ $\mathrm{h}_{3}, \overrightarrow{\mathrm{OP}}=\overrightarrow{\mathrm{p}_{1}}, \overrightarrow{\mathrm{OS}_{\mathrm{jl}}}=\overrightarrow{\mathrm{q}_{\mathrm{j}}}$ 
$\left.\overrightarrow{\mathrm{P}_{\mathrm{J}} \mathrm{S}_{\mathrm{j}}}\right|_{\mathrm{j}=1,2,3}=\left.\overrightarrow{\mathrm{L}_{\mathrm{j}}}\right|_{\mathrm{j}=1,2,3}$

\subsection{Geometry Diagrams of Spatial 3-Dof Parallel Ma- nipulator}

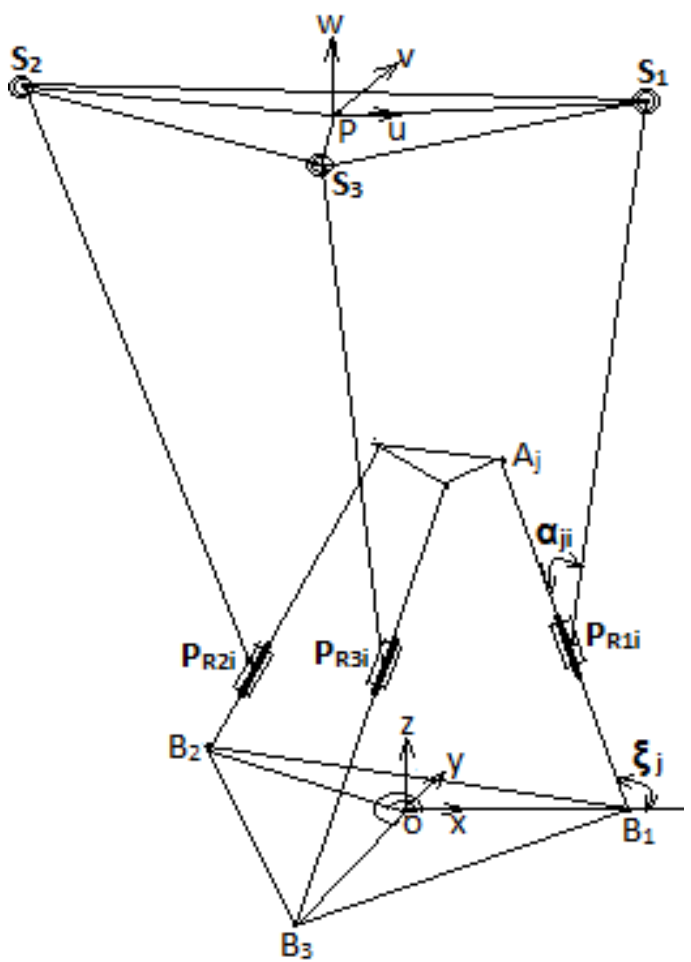

Fig. 1 Spatial 3-dof parallel manipulator
Fig. 2 Geometry of moving platform

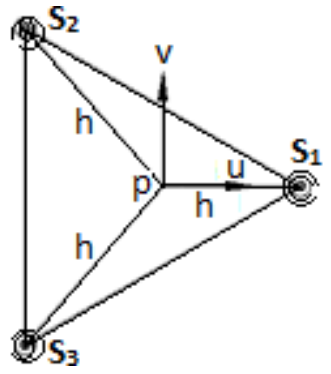

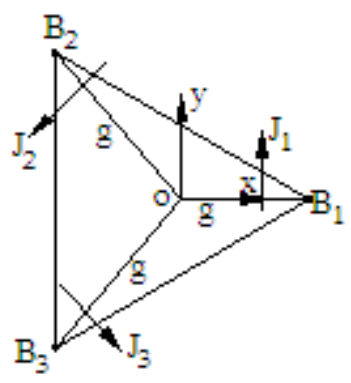

Fig. 3 Geometry of fixed base platform

\subsection{Degree of Freedom of Spatial Parallel Manipula- tor}

The degree of freedom is the number of input parameters required to specify the configuration of a spatial parallel manipulator completely. The equation depends on number of links, number of joints and type of joints incorporated in the selected spatial parallel manipulator. The spatial parallel manipulator consists of eight links, three rotational joints, three sliding joints and three spherical joints. The dof of spatial parallel manipulator can be calculated by using Kutzbach criterion.

$\mathrm{f}=6(\mathrm{n}-1)-5 \mathrm{j}_{1}-4 \mathrm{j}_{2}-3 \mathrm{j}_{3}-2 \mathrm{j}_{4}-\mathrm{j}_{5}=6(8-1)-5 * 6-$

$4 * 0-3 * 3-2 * 0-1 * 0=3$

Where $f$ denotes dof of a spatial parallel manipulator.

$\mathrm{n}$ denotes number of links of a manipulator.

$\mathrm{j}_{1}$ represent the number of links or joints which losses $5 \mathrm{dof}$ $\mathrm{j}_{2}$ represent the number of links or joints which losses $4 \mathrm{dof}$ $j_{3}$ represent the number of links or joints which losses 3 dof $\mathrm{j}_{4}$ represent the number of links or joints which losses 2 dof $\mathrm{j}_{5}$ represent the number of links or joints which losses 1 dof

\section{Forward Kinematic Position Analysis}

First a set of actuated inputs are given, then the position and orientation of the moving platform are obtained by forward kinematic

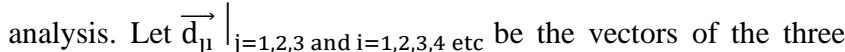
actuated input joint variables along the inclined fixed limbs at an angle of $\xi_{j}$ with respect to $\left.\overrightarrow{\mathrm{OB}}_{j}\right|_{j=1,2,3}$ and $\left[\begin{array}{llllll}\mathrm{P}_{\mathrm{xi}} & \mathrm{P}_{\mathrm{yi}} & \mathrm{P}_{\mathrm{zi}} & \psi_{\mathrm{i}} & \theta_{\mathrm{i}} & \phi_{\mathrm{i}}\end{array}\right]^{\mathrm{T}}$ are the vectors of constrained and unconstrained Cartesian variables which describes the position and orientation of the moving platform with respect to fixed coordinate system. Let $\left.\alpha_{\mathrm{ji}}\right|_{\mathrm{j}=1,2,3 \text { and } \mathrm{i}=1,2,3,4 \text { etc }}$ be the angle made by the $i^{\text {th }}$ position of the $\left.\operatorname{limb} \overrightarrow{\mathrm{P}_{\mathrm{R}} \mathrm{S}_{\mathrm{j}}}\right|_{j=1,2,3}$ with respect to $i^{\text {th }}$ actuation along the inclined fixed limb i.e. $\left.\overrightarrow{B_{j} A_{j}}\right|_{j=1,2,3}$, then the position vector of spherical joint $\left.\overrightarrow{\mathrm{OS}_{j}}\right|_{j=1,2,3}$ with respect to the fixed coordinate system can be expressed as

$\overrightarrow{\mathrm{q}_{\mathrm{j}}}=\left[\left(\mathrm{g}_{\mathrm{j}}+\mathrm{d}_{\mathrm{ji}} \cos \xi_{\mathrm{j}}+\mathrm{L}_{\mathrm{j}} \cos \left(\xi_{\mathrm{j}}-\alpha_{\mathrm{ji}}\right)\right) \cos \beta_{\mathrm{j}}\right] \mathrm{i}+\left[\left(\mathrm{g}_{\mathrm{j}}+\right.\right.$ $\left.\left.\mathrm{d}_{\mathrm{ji}} \cos \xi_{\mathrm{j}}+\mathrm{L}_{\mathrm{j}} \cos \left(\xi_{\mathrm{j}}-\alpha_{\mathrm{ji}}\right)\right) \sin \beta_{\mathrm{j}}\right] \mathrm{j}+\left[\mathrm{d}_{\mathrm{ji}} \sin \xi_{\mathrm{j}}+\mathrm{L}_{\mathrm{j}} \sin \left(\xi_{\mathrm{j}}-\right.\right.$ $\left.\left.\alpha_{\mathrm{ji}}\right)\right] \mathrm{k}$

Where $\mathrm{j}=1,2,3$ and $\mathrm{i}=1,2,3,4$ etc

Consider at $\left.\beta_{\mathrm{j}}\right|_{\mathrm{j}=1,2,3}$ are $0^{0}, 120^{\circ}, 240^{\circ}$ and $\mathrm{g}_{1}=\mathrm{g}_{2}=\mathrm{g}_{3}=$ $\mathrm{g}, \mathrm{L}_{1}=\mathrm{L}_{2}=\mathrm{L}_{3}=\mathrm{L}, \xi_{1}=\xi_{2}=\xi_{3}=\xi$ then Eq. (2) can be written as

$\overrightarrow{\mathrm{q}_{11}}=\left[\left(\mathrm{g}+\mathrm{d}_{1 \mathrm{i}} \cos \xi+\mathrm{L} \cos \left(\xi-\alpha_{1 \mathrm{i}}\right)\right) \cos \beta_{1}\right] \mathrm{i}+[(\mathrm{g}+$ $\left.\left.d_{1 i} \cos \xi+L \cos \left(\xi-\alpha_{1 i}\right)\right) \sin \beta_{1}\right] j+\left[d_{1 i} \sin \xi+L \sin \left(\xi-\alpha_{1 i}\right)\right] k$

$\overrightarrow{\mathrm{q}_{21}}=\left[\left(\mathrm{g}+\mathrm{d}_{2 \mathrm{i}} \cos \xi+\mathrm{L} \cos \left(\xi-\alpha_{2 \mathrm{i}}\right)\right) \cos \beta_{2}\right] \mathrm{i}+[(\mathrm{g}+$

$\left.\left.d_{2 \mathrm{i}} \cos \xi+L \cos \left(\xi-\alpha_{2 \mathrm{i}}\right)\right) \sin \beta_{2}\right] \mathrm{j}+\left[\mathrm{d}_{2 \mathrm{i}} \sin \xi+\mathrm{L} \sin \left(\xi-\alpha_{2 \mathrm{i}}\right)\right] \mathrm{k}$

$\overrightarrow{\mathrm{q}_{31}}=\left[\left(\mathrm{g}+\mathrm{d}_{3 \mathrm{i}} \cos \xi+\mathrm{L} \cos \left(\xi-\alpha_{3 \mathrm{i}}\right)\right) \cos \beta_{3}\right] \mathrm{i}+[(\mathrm{g}+$

$\left.\left.d_{3 i} \cos \xi+L \cos \left(\xi-\alpha_{3 i}\right)\right) \sin \beta_{3}\right] j+\left[d_{3 i} \sin \xi+L \sin \left(\xi-\alpha_{3 i}\right)\right] k$

The position and orientation of the limbs can be determined by the geometric distance between two spherical joints that are to be constant in magnitude. i.e.

$\left|\overrightarrow{\mathrm{S}_{1} \mathrm{~S}_{2}}\right|=\left|\overrightarrow{\mathrm{S}_{2} \mathrm{~S}_{3}}\right|=\left|\overrightarrow{\mathrm{S}_{3} \mathrm{~S}_{1}}\right|=\sqrt{3} \mathrm{~h}$

$\Rightarrow\left|\overrightarrow{\mathrm{q}_{11}}-\overrightarrow{\mathrm{q}_{21}}\right|=\left|\overrightarrow{\mathrm{q}_{21}}-\overrightarrow{\mathrm{q}_{31}}\right|=\left|\overrightarrow{\mathrm{q}_{31}}-\overrightarrow{\mathrm{q}_{11}}\right|=\sqrt{3 \mathrm{~h}}$

Subtracting Eq. (4) from Eq. (3) then

$\overrightarrow{\mathrm{q}_{11}}-\overrightarrow{\mathrm{q}_{21}}=\left[\left(\left(\mathrm{a}_{12}-1\right) \mathrm{g}+\left(\mathrm{a}_{12} \mathrm{~d}_{1 \mathrm{i}}-\mathrm{d}_{2 \mathrm{i}}\right) \cos \xi+\mathrm{L}\left(\mathrm{a}_{12} \cos (\xi-\right.\right.\right.$ $\left.\left.\left.\left.\alpha_{1 \mathrm{i}}\right)-\cos \left(\xi-\alpha_{2 \mathrm{i}}\right)\right)\right) \cos \beta_{2}\right] \mathrm{i}+\left[\left(\left(\mathrm{b}_{12}-1\right) \mathrm{g}+\left(\mathrm{b}_{12} \mathrm{~d}_{1 \mathrm{i}}-\right.\right.\right.$ $\left.\left.\left.\mathrm{d}_{2 \mathrm{i}}\right) \cos \xi+\mathrm{L}\left(\mathrm{b}_{12} \cos \left(\xi-\alpha_{1 \mathrm{i}}\right)-\cos \left(\xi-\alpha_{2 \mathrm{i}}\right)\right)\right) \sin \beta_{2}\right] \mathrm{j}+$ $\left[\left(\mathrm{d}_{1 \mathrm{i}}-\mathrm{d}_{2 \mathrm{i}}\right) \sin \xi+\mathrm{L}\left(\sin \left(\xi-\alpha_{1 \mathrm{i}}\right)-\sin \left(\xi-\alpha_{2 \mathrm{i}}\right)\right)\right] \mathrm{k}$

Subtracting Eq. (5) from Eq. (4) then

$\overrightarrow{\mathrm{q}_{21}}-\overrightarrow{\mathrm{q}_{31}}=\left[\left(\left(\mathrm{a}_{23}-1\right) \mathrm{g}+\left(\mathrm{a}_{23} \mathrm{~d}_{2 \mathrm{i}}-\mathrm{d}_{3 \mathrm{i}}\right) \cos \xi+\mathrm{L}\left(\mathrm{a}_{23} \cos (\xi-\right.\right.\right.$ $\left.\left.\left.\left.\alpha_{2 \mathrm{i}}\right)-\cos \left(\xi-\alpha_{3 \mathrm{i}}\right)\right)\right) \cos \beta_{3}\right] \mathrm{i}+\left[\left(\left(\mathrm{b}_{23}-1\right) \mathrm{g}+\left(\mathrm{b}_{23} \mathrm{~d}_{2 \mathrm{i}}-\right.\right.\right.$ $\left.\left.\left.\mathrm{d}_{3 \mathrm{i}}\right) \cos \xi+\mathrm{L}\left(\mathrm{b}_{23} \cos \left(\xi-\alpha_{2 \mathrm{i}}\right)-\cos \left(\xi-\alpha_{3 \mathrm{i}}\right)\right)\right) \sin \beta_{3}\right] \mathrm{j}+$ $\left[\left(d_{2 \mathrm{i}}-d_{3 \mathrm{i}}\right) \sin \xi+\mathrm{L}\left(\sin \left(\xi-\alpha_{2 \mathrm{i}}\right)-\sin \left(\xi-\alpha_{3 \mathrm{i}}\right)\right)\right] \mathrm{k}$

Subtracting Eq. (5) from Eq. (3) then

$\overrightarrow{\mathrm{q}_{11}}-\overrightarrow{\mathrm{q}_{31}}=\left[\left(\left(\mathrm{a}_{13}-1\right) \mathrm{g}+\left(\mathrm{a}_{13} \mathrm{~d}_{1 \mathrm{i}}-\mathrm{d}_{3 \mathrm{i}}\right) \cos \xi+\mathrm{L}\left(\mathrm{a}_{13} \cos (\xi-\right.\right.\right.$ $\left.\left.\left.\left.\alpha_{1 \mathrm{i}}\right)-\cos \left(\xi-\alpha_{3 \mathrm{i}}\right)\right)\right) \cos \beta_{3}\right] \mathrm{i}+\left[\left(\left(\mathrm{b}_{13}-1\right) \mathrm{g}+\left(\mathrm{b}_{13} \mathrm{~d}_{1 \mathrm{i}}-\right.\right.\right.$ 
$\left.\left.\left.d_{3 i}\right) \cos \xi+L\left(b_{13} \cos \left(\xi-\alpha_{1 i}\right)-\cos \left(\xi-\alpha_{3 i}\right)\right)\right) \sin \beta_{3}\right] j+$

$\left[\left(d_{1 \mathrm{i}}-\mathrm{d}_{3 \mathrm{i}}\right) \sin \xi+\mathrm{L}\left(\sin \left(\xi-\alpha_{1 \mathrm{i}}\right)-\sin \left(\xi-\alpha_{3 \mathrm{i}}\right)\right)\right] \mathrm{k}$

Eq. (7) can be written as

$\overrightarrow{\mathrm{q}_{11}}-\overrightarrow{\mathrm{q}_{21}}=\left[\mathrm{a}_{12 \mathrm{i}} \mathrm{L} \cos \beta_{2} \cos \xi+\mathrm{L}\left(\mathrm{a}_{12} \cos \left(\xi-\alpha_{1 \mathrm{i}}\right)-\right.\right.$

$\left.\left.\cos \left(\xi-\alpha_{2 \mathrm{i}}\right)\right) \cos \beta_{2}\right] \mathrm{i}+\left[\mathrm{b}_{12 \mathrm{i}} \mathrm{L} \sin \beta_{2} \cos \xi+\mathrm{L}\left(\mathrm{b}_{12} \cos (\xi-\right.\right.$

$\left.\left.\left.\alpha_{1 \mathrm{i}}\right)-\cos \left(\xi-\alpha_{2 \mathrm{i}}\right)\right) \sin \beta_{2}\right] \mathrm{j}+\left[\mathrm{d}_{12 \mathrm{i}} \mathrm{L} \cos \xi+\mathrm{L}\left(\sin \left(\xi-\alpha_{1 \mathrm{i}}\right)-\right.\right.$

$\left.\left.\sin \left(\xi-\alpha_{2 \mathrm{i}}\right)\right)\right] \mathrm{k}$

Eq. (8) can be written as

$\overrightarrow{\mathrm{q}_{21}}-\overrightarrow{\mathrm{q}_{31}}=\left[\mathrm{a}_{23 \mathrm{i}} \mathrm{L} \cos \beta_{3} \cos \xi+\mathrm{L}\left(\mathrm{a}_{23} \cos \left(\xi-\alpha_{2 \mathrm{i}}\right)-\right.\right.$

$\left.\left.\cos \left(\xi-\alpha_{3 \mathrm{i}}\right)\right) \cos \beta_{3}\right] \mathrm{i}+\left[\mathrm{b}_{23 \mathrm{i}} \mathrm{L} \sin \beta_{3} \cos \xi+\mathrm{L}\left(\mathrm{b}_{23} \cos (\xi-\right.\right.$

$\left.\left.\left.\alpha_{2 \mathrm{i}}\right)-\cos \left(\xi-\alpha_{3 \mathrm{i}}\right)\right) \sin \beta_{3}\right] \mathrm{j}+\left[\mathrm{d}_{23 \mathrm{i}} \mathrm{L} \cos \xi+\mathrm{L}\left(\sin \left(\xi-\alpha_{2 \mathrm{i}}\right)-\right.\right.$

$\left.\left.\sin \left(\xi-\alpha_{3 \mathrm{i}}\right)\right)\right] \mathrm{k}$

Eq. (9) can be written as

$\overrightarrow{\mathrm{q}_{11}}-\overrightarrow{\mathrm{q}_{31}}=\left[\mathrm{a}_{13 \mathrm{i}} \mathrm{L} \cos \beta_{3} \cos \xi+\mathrm{L}\left(\mathrm{a}_{13} \cos \left(\xi-\alpha_{1 \mathrm{i}}\right)-\right.\right.$

$\left.\left.\cos \left(\xi-\alpha_{3 i}\right)\right) \cos \beta_{3}\right] i+\left[b_{13 i} L \sin \beta_{3} \cos \xi+L\left(b_{13} \cos (\xi-\right.\right.$

$\left.\left.\left.\alpha_{1 \mathrm{i}}\right)-\cos \left(\xi-\alpha_{3 \mathrm{i}}\right)\right) \sin \beta_{3}\right] \mathrm{j}+\left[\mathrm{d}_{13 \mathrm{i}} \mathrm{L} \cos \xi+\mathrm{L}\left(\sin \left(\xi-\alpha_{1 \mathrm{i}}\right)-\right.\right.$

$\left.\left.\sin \left(\xi-\alpha_{3 \mathrm{i}}\right)\right)\right] \mathrm{k}$

By substituting Eq. (10) in Eq. (6) then

$\mathrm{C}_{1 \mathrm{i}}^{\prime} \sin ^{2} \alpha_{1 \mathrm{i}}+\mathrm{C}_{2 \mathrm{i}}^{\prime} \cos ^{2} \alpha_{1 \mathrm{i}}+\mathrm{C}_{3 \mathrm{i}}^{\prime} \sin ^{2} \alpha_{2 \mathrm{i}}+\mathrm{C}_{4 \mathrm{i}}^{\prime} \cos ^{2} \alpha_{2 \mathrm{i}}+$

$\mathrm{C}_{5 \mathrm{i}}^{\prime} \sin \alpha_{1 \mathrm{i}} \cos \alpha_{1 \mathrm{i}}+\mathrm{C}_{6 \mathrm{i}}^{\prime} \sin \alpha_{1 \mathrm{i}} \sin \alpha_{2 \mathrm{i}}+\mathrm{C}_{7 \mathrm{i}}^{\prime} \sin \alpha_{1 \mathrm{i}} \cos \alpha_{2 \mathrm{i}}+$

$\mathrm{C}_{8 \mathrm{i}}^{\prime} \cos \alpha_{1 \mathrm{i}} \sin \alpha_{2 \mathrm{i}}+\mathrm{C}_{9 \mathrm{i}}^{\prime} \cos \alpha_{1 \mathrm{i}} \cos \alpha_{2 \mathrm{i}}+\mathrm{C}_{10 \mathrm{i}}^{\prime} \sin \alpha_{2 \mathrm{i}} \cos \alpha_{2 \mathrm{i}}+$

$C_{11 \mathrm{i}}^{\prime} \sin \alpha_{1 \mathrm{i}}+\mathrm{C}_{12 \mathrm{i}}^{\prime} \cos \alpha_{1 \mathrm{i}}+\mathrm{C}_{13 \mathrm{i}}^{\prime} \sin \alpha_{2 \mathrm{i}}+\mathrm{C}_{14 \mathrm{i}}^{\prime} \cos \alpha_{2 \mathrm{i}}+\mathrm{C}_{15 \mathrm{i}}^{\prime}=0$

By substituting Eq. (11) in Eq. (6) then

$\mathrm{C}_{1 \mathrm{i}}^{\prime \prime} \sin ^{2} \alpha_{2 \mathrm{i}}+\mathrm{C}_{2 \mathrm{i}}^{\prime \prime} \cos ^{2} \alpha_{2 \mathrm{i}}+\mathrm{C}_{3 \mathrm{i}}^{\prime \prime} \sin ^{2} \alpha_{3 \mathrm{i}}+\mathrm{C}_{4 \mathrm{i}}^{\prime \prime} \cos ^{2} \alpha_{3 \mathrm{i}}+$

$\mathrm{C}_{5 \mathrm{i}}^{\prime \prime} \sin \alpha_{2 \mathrm{i}} \cos \alpha_{2 \mathrm{i}}+\mathrm{C}_{6 \mathrm{i}}^{\prime \prime} \sin \alpha_{2 \mathrm{i}} \sin \alpha_{3 \mathrm{i}}+\mathrm{C}_{7 \mathrm{i}}^{\prime \prime} \sin \alpha_{2 \mathrm{i}} \cos \alpha_{3 \mathrm{i}}+$

$\mathrm{C}_{8 \mathrm{i}}^{\prime \prime} \cos \alpha_{2 \mathrm{i}} \sin \alpha_{3 \mathrm{i}}+\mathrm{C}_{9 \mathrm{i}}^{\prime \prime} \cos \alpha_{2 \mathrm{i}} \cos \alpha_{3 \mathrm{i}}+\mathrm{C}_{10 \mathrm{i}}^{\prime \prime} \sin \alpha_{3 \mathrm{i}} \cos \alpha_{3 \mathrm{i}}+$

$\mathrm{C}_{11 \mathrm{i}}^{\prime \prime} \sin \alpha_{2 \mathrm{i}}+\mathrm{C}_{12 \mathrm{i}}^{\prime \prime} \cos \alpha_{2 \mathrm{i}}+\mathrm{C}_{13 \mathrm{i}}^{\prime \prime} \sin \alpha_{3 \mathrm{i}}+\mathrm{C}_{14 \mathrm{i}}^{\prime \prime} \cos \alpha_{3 \mathrm{i}}+\mathrm{C}_{15 \mathrm{i}}^{\prime \prime}=$

0

By substituting Eq. (12) in Eq. (6) then

$\mathrm{C}_{1 \mathrm{i}}^{\prime \prime \prime} \sin ^{2} \alpha_{1 \mathrm{i}}+\mathrm{C}_{2 \mathrm{i}}^{\prime \prime \prime} \cos ^{2} \alpha_{1 \mathrm{i}}+\mathrm{C}_{3 \mathrm{i}}^{\prime \prime \prime} \sin ^{2} \alpha_{3 \mathrm{i}}+\mathrm{C}_{4 \mathrm{i}}^{\prime \prime \prime} \cos ^{2} \alpha_{3 \mathrm{i}}+$ $\mathrm{C}_{5 \mathrm{i}}^{\prime \prime \prime} \sin \alpha_{1 \mathrm{i}} \cos \alpha_{1 \mathrm{i}}+\mathrm{C}_{6 \mathrm{i}}^{\prime \prime \prime} \sin \alpha_{1 \mathrm{i}} \sin \alpha_{3 \mathrm{i}}+\mathrm{C}_{7 \mathrm{i}}^{\prime \prime \prime} \sin \alpha_{1 \mathrm{i}} \cos \alpha_{3 \mathrm{i}}+$ $\mathrm{C}_{8 \mathrm{i}}^{\prime \prime \prime} \cos \alpha_{1 \mathrm{i}} \sin \alpha_{3 \mathrm{i}}+\mathrm{C}_{9 \mathrm{i}}^{\prime \prime \prime} \cos \alpha_{1 \mathrm{i}} \cos \alpha_{3 \mathrm{i}}+\mathrm{C}_{10 \mathrm{i}}^{\prime \prime \prime} \sin \alpha_{3 \mathrm{i}} \cos \alpha_{3 \mathrm{i}}+$ $\mathrm{C}_{11 \mathrm{i}}^{\prime \prime \prime} \sin \alpha_{1 \mathrm{i}}+\mathrm{C}_{12 \mathrm{i}}^{\prime \prime \prime} \cos \alpha_{1 \mathrm{i}}+\mathrm{C}_{13 \mathrm{i}}^{\prime \prime \prime} \sin \alpha_{3 \mathrm{i}}+\mathrm{C}_{14 \mathrm{i}}^{\prime \prime \prime} \cos \alpha_{3 \mathrm{i}}+\mathrm{C}_{15 \mathrm{i}}^{\prime \prime \prime}=0$

Constants are given in appendix

In the Eq. (13), Eq. (14) and Eq. (15) g, L, $\xi, \beta$ are the given values and $d_{1 i}, d_{2 i}, d_{3 i}$ are the assumed $i^{\text {th }}$ actuated values of the three successive prismatic actuators, then $c_{1 \mathrm{i}}^{\prime}$ to $c_{15 \mathrm{i}}^{\prime}, \mathrm{c}_{1 \mathrm{i}}^{\prime \prime}$ to $\mathrm{c}_{15 \mathrm{i}}^{\prime \prime}$ and $\mathrm{c}_{1 \mathrm{i}}^{\prime \prime \prime}$ to $\mathrm{c}_{15 \mathrm{i}}^{\prime \prime \prime}$ are the constant values, which depends on the above given and assumed values. The Eq. (13), Eq. (14) and Eq. (15) contain trigonometric ratios i.e. sine and cosine of two degree polynomial with 15 terms and three unknown ues $\alpha_{1 \mathrm{i}}, \alpha_{2 \mathrm{i}}, \alpha_{3 \mathrm{i}}$ for each actuation. By solving Eq. (13), Eq. (14) and Eq. (15) for all $i^{\text {th }}$ actuations the values of three unknowns $\alpha_{1 \mathrm{i}}, \alpha_{2 \mathrm{i}}, \alpha_{3 \mathrm{i}}$ are to be determined and from the above calculated values, $\overrightarrow{\mathrm{q}_{11}}, \overrightarrow{\mathrm{q}_{21}}, \overrightarrow{\mathrm{q}_{31}}$ can to be determined. From the geometry of the structure of the manipulator

$$
\begin{aligned}
& \overrightarrow{\mathrm{p}_{1}}=\overrightarrow{\mathrm{q}_{11}}-\mathrm{o}_{\mathrm{R}_{\mathrm{p}_{\mathrm{i}}}} \overrightarrow{\mathrm{h}_{1}} \\
& \overrightarrow{\mathrm{p}_{1}}=\overrightarrow{\mathrm{q}_{21}}-\mathrm{o}_{\mathrm{R}_{\mathrm{p}_{\mathrm{i}}}} \overrightarrow{\mathrm{h}_{2}} \\
& \overrightarrow{\mathrm{p}_{1}}=\overrightarrow{\mathrm{q}_{31}}-\mathrm{o}_{\mathrm{R}_{\mathrm{p}_{\mathrm{i}}}} \overrightarrow{\mathrm{h}_{3}}
\end{aligned}
$$

That means

$$
\begin{aligned}
& \overrightarrow{\mathrm{p}_{1}}= \\
& \frac{1}{3}\left(\overrightarrow{\mathrm{q}_{11}}+\overrightarrow{\mathrm{q}_{21}}+\overrightarrow{\mathrm{q}_{31}}\right)- \\
& \frac{1}{3} \mathrm{o}_{\mathrm{R}_{\mathrm{p}_{\mathrm{i}}}} \mathrm{h}\left[\cos \beta_{1}+\cos \beta_{2}+\cos \beta_{3} \quad \sin \beta_{1}+\sin \beta_{2}+\sin \beta_{3} \quad 0\right]^{\mathrm{T}}
\end{aligned}
$$

Consider

$$
\overrightarrow{q_{1231}}=\left(\overrightarrow{q_{11}}+\overrightarrow{q_{21}}+\overrightarrow{q_{31}}\right)=q_{123 x i} \hat{i}+q_{123 y i} \hat{\jmath}+q_{123 z i} \hat{k}
$$$$
\cos \beta_{123}=\cos \beta_{1}+\cos \beta_{2}+\cos \beta_{3}
$$

$\sin \beta_{123}=\sin \beta_{1}+\sin \beta_{2}+\sin \beta_{3}$

Then the Eq. (16) can be written as

$\left[\begin{array}{l}p_{x i} \\ p_{y i} \\ p_{z i}\end{array}\right]=\frac{1}{3}\left[\begin{array}{l}q_{123 x i} \\ q_{123 y i} \\ q_{123 z i}\end{array}\right]-\frac{h}{3}\left[\begin{array}{lll}u_{x i} & v_{x i} & w_{x i} \\ u_{y i} & v_{y i} & w_{y i} \\ u_{z i} & v_{z i} & w_{z i}\end{array}\right]\left[\begin{array}{c}\cos \beta_{123} \\ \sin \beta_{123} \\ 0\end{array}\right]$

$\left[\begin{array}{l}p_{x i} \\ p_{y i} \\ p_{z i}\end{array}\right]=\left[\begin{array}{c}\frac{h}{2 \cos \phi_{i}}\left(\cos \theta_{i}-\cos \psi_{\mathrm{i}} \cos 2 \phi_{\mathrm{i}}\right) \\ -\mathrm{h} \cos \psi_{\mathrm{i}} \sin \phi_{\mathrm{i}} \\ \mathrm{p}_{\mathrm{zi}}\end{array}\right]$

$\left[\begin{array}{lll}u_{x} & v_{x} & w_{x} \\ u_{y} & v_{y} & w_{y} \\ u_{z} & v_{z} & w_{z}\end{array}\right]=\left[\begin{array}{ccc}c \theta_{i} c \phi_{i}+s \psi_{i} s \theta_{i} s \phi_{i} & -c \theta_{i} s \phi_{i}+s \psi_{i} s \theta_{i} c \phi_{i} & s \theta_{i} c \psi_{i} \\ c \psi_{i} s \phi_{i} & c \psi_{i} c \phi_{i} & -s \psi_{i} \\ -s \theta_{i} c \phi_{i}+s \psi_{i} c \theta_{i} s \phi_{i} & s \theta_{i} s \phi_{i}+s \psi_{i} c \theta_{i} c \phi_{i} & c \theta_{i} c \psi_{i}\end{array}\right]$

From Eq. (17)

$\frac{3 \mathrm{~h}}{2 \cos \phi_{\mathrm{i}}}\left(\cos \theta_{\mathrm{i}}-\cos \psi_{\mathrm{i}} \cos 2 \phi_{\mathrm{i}}\right)=\mathrm{q}_{123 x \mathrm{i}}-\left(\cos \theta_{\mathrm{i}} \cos \phi_{\mathrm{i}}+\right.$

$\left.\sin \psi_{\mathrm{i}} \sin \theta_{\mathrm{i}} \sin \phi_{\mathrm{i}}\right) \mathrm{h} \cos \beta_{123}-$

$\left(-\cos \theta_{\mathrm{i}} \sin \phi_{\mathrm{i}}+\sin \psi_{\mathrm{i}} \sin \theta_{\mathrm{i}} \cos \phi_{\mathrm{i}}\right) \mathrm{h} \sin \beta_{123}$

$-3 \mathrm{~h} \cos \psi_{\mathrm{i}} \sin \phi_{\mathrm{i}}=\mathrm{q}_{123 \mathrm{yi}}-\cos \psi_{\mathrm{i}} \sin \phi_{\mathrm{i}} \mathrm{h} \cos \beta_{123}-$

$\cos \psi_{\mathrm{i}} \cos \phi_{\mathrm{i}} \mathrm{h} \sin \beta_{123}$

Where

$$
\sin \phi_{i}=\frac{\sin \psi_{i} \sin \theta_{i}}{1+\cos \theta_{i} \cos \psi_{i}}
$$

The Eq. (19) can be written as

$\left(\mathrm{h} \sin \beta_{123}\right) \cos ^{2} \psi_{\mathrm{i}}-\left(\mathrm{q}_{123 \mathrm{yi}}-\mathrm{h} \sin \beta_{123}\right) \cos \psi_{\mathrm{i}} \cos \theta_{\mathrm{i}}+$

$\left(\cos \beta_{123}-3\right) h \cos \psi_{\mathrm{i}} \sin \psi_{\mathrm{i}} \sin \theta_{\mathrm{i}}-\mathrm{q}_{123 \mathrm{yi}}=0$

The Eq. (18) can be written as

$\left(2 \cos \beta_{123}+6\right) h \cos ^{3} \psi_{\mathrm{i}}+\left(6 \mathrm{~h}+2 \mathrm{q}_{123 \times \mathrm{i}}\right) \cos ^{2} \psi_{\mathrm{i}} \cos \theta_{\mathrm{i}}+$ $\left(2 \mathrm{q}_{123 \times \mathrm{i}}-2 \mathrm{~h} \cos \beta_{123}\right) \cos \psi_{\mathrm{i}} \cos ^{2} \theta_{\mathrm{i}}+\left(2 \mathrm{q}_{123 \times \mathrm{i}}-\right.$

$\left.2 \mathrm{~h} \cos \beta_{123}-3 \mathrm{~h}\right)\left(\cos \psi_{\mathrm{i}}+\cos \theta_{\mathrm{i}}\right)-\left(3+2 \cos \beta_{123}\right)\left(\cos \psi_{\mathrm{i}}+\right.$ $\left.\cos \theta_{\mathrm{i}}\right) \mathrm{h} \cos ^{2} \psi_{\mathrm{i}} \cos ^{2} \theta_{\mathrm{i}}-$

$\left(2 \mathrm{~h} \sin \beta_{123}\right)\left(\cos \psi_{\mathrm{i}}+\cos \theta_{\mathrm{i}}\right) \cos \psi_{\mathrm{i}} \sin \psi_{\mathrm{i}} \sin \theta_{\mathrm{i}}=0$

The Eq. (20) contains $3^{\text {rd }}$ degree polynomial with four terms and the Eq. (21) contains $5^{\text {th }}$ degree polynomial with six terms and two unknowns for $\mathrm{i}^{\text {th }}$ number of actuations. For each actuation the values of $\theta_{\mathrm{i}}$ and $\psi_{\mathrm{i}}$ can be determined.

From $\sin \phi_{i}=\frac{\sin \psi_{i} \sin \theta_{i}}{1+\cos \theta_{i} \cos \psi_{i}}$ for each actuation the values of $\phi_{i}$ can be calculated.

From Eq. (17) the value of

$3 \mathrm{p}_{\mathrm{zi}}=\mathrm{q}_{123 \mathrm{zi}}+\left(\sin \theta_{\mathrm{i}} \cos \phi_{\mathrm{i}}-\sin \psi_{\mathrm{i}} \cos \theta_{\mathrm{i}} \sin \phi_{\mathrm{i}}\right) \mathrm{h} \cos \beta_{123}-$ $\left(\sin \theta_{\mathrm{i}} \sin \phi_{\mathrm{i}}+\sin \psi_{\mathrm{i}} \cos \theta_{\mathrm{i}} \cos \phi_{\mathrm{i}}\right) \mathrm{h} \sin \beta_{123}$

By substituting the calculated values of $\theta_{i}, \psi_{i}$ and $\phi_{i}$ in Eq. (22), the values of $\mathrm{p}_{\mathrm{zi}}$ can be determined. Therefore for each prismatic actuation of the limb, the position and orientation of the moving manipulator constrained variables $\mathrm{p}_{\mathrm{xi}}, \mathrm{p}_{\mathrm{yi}}, \phi_{\mathrm{i}}$ and unconstrained variables $\theta_{\mathrm{i}}, \psi_{\mathrm{i}}, \mathrm{p}_{\mathrm{zi}}$ are to be calculated.

\section{Conclusion}

In this paper the forward position kinematic analysis of spatial 3 dof parallel manipulator are analyzed. The dof of spatial parallel manipulator is calculated by using kutzbach criterion. The physical constraints followed by Euler angle representation are introduced along the plane of spherical joints, actuated sliding joints and limbs are considered. The forward position kinematic problem is solved through geometric method by using vector technique. The purpose of this analysis is to develop an analytical method and systematic design procedure to analyze the basic kinematics 
of the manipulator, to check the required and obtained positions of the moving platform of the developed spatial parallel manipulator.

\section{Appendix}

The constants in forward kinematic equation are

$\mathrm{C}_{1 \mathrm{i}}^{\prime}=\mathrm{L}^{2}\left(\mathrm{a}_{12}^{2} \cos ^{2} \beta_{2} \sin ^{2} \xi+\mathrm{b}_{12}^{2} \sin ^{2} \beta_{2} \sin ^{2} \xi+\cos ^{2} \xi\right)$

$\mathrm{C}_{2 \mathrm{i}}^{\prime}=\mathrm{L}^{2}\left(\mathrm{a}_{12}^{2} \cos ^{2} \beta_{2} \cos ^{2} \xi+\mathrm{b}_{12}^{2} \sin ^{2} \beta_{2} \cos ^{2} \xi+\sin ^{2} \xi\right)$

$\mathrm{C}_{3 \mathrm{i}}^{\prime}=\mathrm{L}^{2}\left(\cos ^{2} \beta_{2} \sin ^{2} \xi+\sin ^{2} \beta_{2} \sin ^{2} \xi+\cos ^{2} \xi\right)$

$\mathrm{C}_{4 \mathrm{i}}^{\prime}=\mathrm{L}^{2}\left(\cos ^{2} \beta_{2} \cos ^{2} \xi+\sin ^{2} \beta_{2} \cos ^{2} \xi+\sin ^{2} \xi\right)$

$C_{5 i}^{\prime}=L^{2} \sin 2 \xi\left(a_{12}^{2} \cos ^{2} \beta_{2}+b_{12}^{2} \sin ^{2} \beta_{2}-1\right)$

$C_{6 i}^{\prime}=-2 L^{2}\left(a_{12} \cos ^{2} \beta_{2} \sin ^{2} \xi+b_{12} \sin ^{2} \beta_{2} \sin ^{2} \xi+\cos ^{2} \xi\right)$

$\mathrm{C}_{7 \mathrm{i}}^{\prime}=-\mathrm{L}^{2} \sin 2 \xi\left(\mathrm{a}_{12} \cos ^{2} \beta_{2}+\mathrm{b}_{12} \sin ^{2} \beta_{2}-1\right)$

$\mathrm{C}_{8 \mathrm{i}}^{\prime}=-\mathrm{L}^{2} \sin 2 \xi\left(\mathrm{a}_{12} \cos ^{2} \beta_{2}+\mathrm{b}_{12} \sin ^{2} \beta_{2}-1\right)$

$C_{9 i}^{\prime}=-2 L^{2}\left(a_{12} \cos ^{2} \beta_{2} \cos ^{2} \xi+b_{12} \sin ^{2} \beta_{2} \cos ^{2} \xi+\sin ^{2} \xi\right)$

$\mathrm{C}_{10 \mathrm{i}}^{\prime}=\mathrm{L}^{2} \sin 2 \xi\left(\cos ^{2} \beta_{2}+\sin ^{2} \beta_{2}-1\right)$

$\mathrm{C}_{11 \mathrm{i}}^{\prime}=\mathrm{L}^{2} \sin 2 \xi\left(\mathrm{a}_{12} \mathrm{a}_{12 \mathrm{i}} \cos ^{2} \beta_{2}+\mathrm{b}_{12} \mathrm{~b}_{12 \mathrm{i}} \sin ^{2} \beta_{2}-\mathrm{d}_{12 \mathrm{i}} \cos \xi\right)$

$\mathrm{C}_{12 \mathrm{i}}^{\prime}=2 \mathrm{~L}^{2} \cos \xi\left(\mathrm{a}_{12} \mathrm{a}_{12 \mathrm{i}} \cos ^{2} \beta_{2} \cos \xi+\mathrm{b}_{12} \mathrm{~b}_{12 \mathrm{i}} \sin ^{2} \beta_{2} \cos \xi\right.$ $\left.+d_{12 \mathrm{i}} \sin ^{2} \xi\right)$

$\mathrm{C}_{13 \mathrm{i}}^{\prime}=-\mathrm{L}^{2} \sin 2 \xi\left(\mathrm{a}_{12 \mathrm{i}} \cos ^{2} \beta_{2}+\mathrm{b}_{12 \mathrm{i}} \sin ^{2} \beta_{2}-\mathrm{d}_{12 \mathrm{i}} \cos \xi\right)$

$\mathrm{C}_{14 \mathrm{i}}^{\prime}=-2 \mathrm{~L}^{2} \cos \xi\left(\mathrm{a}_{12 \mathrm{i}} \cos ^{2} \beta_{2} \cos \xi+\mathrm{b}_{12 \mathrm{i}} \sin ^{2} \beta_{2} \cos \xi\right.$ $\left.+\mathrm{d}_{12 \mathrm{i}} \sin ^{2} \xi\right)$

$\mathrm{C}_{15 \mathrm{i}}^{\prime}=\mathrm{L}^{2} \cos ^{2} \xi\left(\mathrm{a}_{12 \mathrm{i}}^{2} \cos ^{2} \beta_{2}+\mathrm{b}_{12 \mathrm{i}}^{2} \sin ^{2} \beta_{2}+\mathrm{d}_{12 \mathrm{i}}^{2} \sin ^{2} \xi\right)-3 \mathrm{~h}^{2}$

$C_{1 i}^{\prime \prime}=L^{2}\left(a_{23}^{2} \cos ^{2} \beta_{3} \sin ^{2} \xi+b_{23}^{2} \sin ^{2} \beta_{3} \sin ^{2} \xi+\cos ^{2} \xi\right)$

$\mathrm{C}_{2 \mathrm{i}}^{\prime \prime}=\mathrm{L}^{2}\left(\mathrm{a}_{23}^{2} \cos ^{2} \beta_{3} \cos ^{2} \xi+\mathrm{b}_{23}^{2} \sin ^{2} \beta_{3} \cos ^{2} \xi+\sin ^{2} \xi\right)$

$\mathrm{C}_{3 \mathrm{i}}^{\prime \prime}=\mathrm{L}^{2}\left(\cos ^{2} \beta_{3} \sin ^{2} \xi+\sin ^{2} \beta_{3} \sin ^{2} \xi+\cos ^{2} \xi\right)$

$\mathrm{C}_{4 \mathrm{i}}^{\prime \prime}=\mathrm{L}^{2}\left(\cos ^{2} \beta_{3} \cos ^{2} \xi+\sin ^{2} \beta_{3} \cos ^{2} \xi+\sin ^{2} \xi\right)$

$C_{5 i}^{\prime \prime}=L^{2} \sin 2 \xi\left(a_{23}^{2} \cos ^{2} \beta_{3}+b_{23}^{2} \sin ^{2} \beta_{3}-1\right)$

$C_{6 i}^{\prime \prime}=-2 L^{2}\left(a_{23} \cos ^{2} \beta_{3} \sin ^{2} \xi+b_{23} \sin ^{2} \beta_{3} \sin ^{2} \xi+\cos ^{2} \xi\right)$

$C_{7 i}^{\prime \prime}=-L^{2} \sin 2 \xi\left(a_{23} \cos ^{2} \beta_{3}+b_{23} \sin ^{2} \beta_{3}-1\right)$

$\mathrm{C}_{8 \mathrm{i}}^{\prime \prime}=-\mathrm{L}^{2} \sin 2 \xi\left(\mathrm{a}_{23} \cos ^{2} \beta_{3}+\mathrm{b}_{23} \sin ^{2} \beta_{3}-1\right)$

$C_{9 i}^{\prime \prime}=-2 L^{2}\left(a_{23} \cos ^{2} \beta_{3} \cos ^{2} \xi+b_{23} \sin ^{2} \beta_{3} \cos ^{2} \xi+\sin ^{2} \xi\right)$

$\mathrm{C}_{10 \mathrm{i}}^{\prime \prime}=\mathrm{L}^{2} \sin 2 \xi\left(\cos ^{2} \beta_{3}+\sin ^{2} \beta_{3}-1\right)$

$C_{11 i}^{\prime \prime}=L^{2} \sin 2 \xi\left(a_{23} a_{23 i} \cos ^{2} \beta_{3}+b_{23} b_{23 i} \sin ^{2} \beta_{3}-d_{23 i} \cos \xi\right)$

$\mathrm{C}_{12 \mathrm{i}}^{\prime \prime}=2 \mathrm{~L}^{2} \cos \xi\left(\mathrm{a}_{23} \mathrm{a}_{23 \mathrm{i}} \cos ^{2} \beta_{3} \cos \xi+\mathrm{b}_{23} \mathrm{~b}_{23 \mathrm{i}} \sin ^{2} \beta_{3} \cos \xi\right.$ $\left.+d_{23 i} \sin ^{2} \xi\right)$

$C_{13 i}^{\prime \prime}=-L^{2} \sin 2 \xi\left(a_{23 i} \cos ^{2} \beta_{3}+b_{23 i} \sin ^{2} \beta_{3}-d_{23 i} \cos \xi\right)$

$\mathrm{C}_{14 \mathrm{i}}^{\prime \prime}=-2 \mathrm{~L}^{2} \cos \xi\left(\mathrm{a}_{23 \mathrm{i}} \cos ^{2} \beta_{3} \cos \xi+\mathrm{b}_{23 \mathrm{i}} \sin ^{2} \beta_{3} \cos \xi\right.$ $\left.+d_{23 \mathrm{i}} \sin ^{2} \xi\right)$

$C_{15 i}^{\prime \prime}=L^{2} \cos ^{2} \xi\left(a_{23 i}^{2} \cos ^{2} \beta_{3}+b_{23 i}^{2} \sin ^{2} \beta_{3}+d_{23 i}^{2} \sin ^{2} \xi\right)-3 h^{2}$

$C_{1 i}^{\prime \prime \prime}=L^{2}\left(a_{13}^{2} \cos ^{2} \beta_{3} \sin ^{2} \xi+b_{13}^{2} \sin ^{2} \beta_{3} \sin ^{2} \xi+\cos ^{2} \xi\right)$

$C_{2 i}^{\prime \prime \prime}=L^{2}\left(a_{13}^{2} \cos ^{2} \beta_{3} \cos ^{2} \xi+b_{13}^{2} \sin ^{2} \beta_{3} \cos ^{2} \xi+\sin ^{2} \xi\right)$

$\mathrm{C}_{3 \mathrm{i}}^{\prime \prime \prime}=\mathrm{L}^{2}\left(\cos ^{2} \beta_{3} \sin ^{2} \xi+\sin ^{2} \beta_{3} \sin ^{2} \xi+\cos ^{2} \xi\right)$

$\mathrm{C}_{4 \mathrm{i}}^{\prime \prime \prime}=\mathrm{L}^{2}\left(\cos ^{2} \beta_{3} \cos ^{2} \xi+\sin ^{2} \beta_{3} \cos ^{2} \xi+\sin ^{2} \xi\right)$

$C_{5 i}^{\prime \prime \prime}=L^{2} \sin 2 \xi\left(a_{13}^{2} \cos ^{2} \beta_{3}+b_{13}^{2} \sin ^{2} \beta_{3}-1\right)$

$C_{6 i}^{\prime \prime \prime}=-2 L^{2}\left(a_{13} \cos ^{2} \beta_{3} \sin ^{2} \xi+b_{13} \sin ^{2} \beta_{3} \sin ^{2} \xi+\cos ^{2} \xi\right)$

$\mathrm{C}_{7 \mathrm{i}}^{\prime \prime \prime}=-\mathrm{L}^{2} \sin 2 \xi\left(\mathrm{a}_{13} \cos ^{2} \beta_{3}+\mathrm{b}_{13} \sin ^{2} \beta_{3}-1\right)$

$\mathrm{C}_{8 \mathrm{i}}^{\prime \prime \prime}=-\mathrm{L}^{2} \sin 2 \xi\left(\mathrm{a}_{13} \cos ^{2} \beta_{3}+\mathrm{b}_{13} \sin ^{2} \beta_{3}-1\right)$

$C_{9 i}^{\prime \prime \prime}=-2 L^{2}\left(a_{13} \cos ^{2} \beta_{3} \cos ^{2} \xi+b_{13} \sin ^{2} \beta_{3} \cos ^{2} \xi+\sin ^{2} \xi\right)$

$\mathrm{C}_{10 \mathrm{i}}^{\prime \prime \prime}=\mathrm{L}^{2} \sin 2 \xi\left(\cos ^{2} \beta_{3}+\sin ^{2} \beta_{3}-1\right)$

$\mathrm{C}_{11 \mathrm{i}}^{\prime \prime \prime}=\mathrm{L}^{2} \sin 2 \xi\left(\mathrm{a}_{13} \mathrm{a}_{13 \mathrm{i}} \cos ^{2} \beta_{3}+\mathrm{b}_{13} \mathrm{~b}_{13 \mathrm{i}} \sin ^{2} \beta_{3}-\mathrm{d}_{13 \mathrm{i}} \cos \xi\right)$

$\mathrm{C}_{12 \mathrm{i}}^{\prime \prime \prime}=2 \mathrm{~L}^{2} \cos \xi\left(\mathrm{a}_{13} \mathrm{a}_{13 \mathrm{i}} \cos ^{2} \beta_{3} \cos \xi+\mathrm{b}_{13} \mathrm{~b}_{13 \mathrm{i}} \sin ^{2} \beta_{3} \cos \xi\right.$ $\left.+d_{13 i} \sin ^{2} \xi\right)$

$\mathrm{C}_{13 \mathrm{i}}^{\prime \prime \prime}=-\mathrm{L}^{2} \sin 2 \xi\left(\mathrm{a}_{13 \mathrm{i}} \cos ^{2} \beta_{3}+\mathrm{b}_{13 \mathrm{i}} \sin ^{2} \beta_{3}-\mathrm{d}_{13 \mathrm{i}} \cos \xi\right)$

$\mathrm{C}_{14 \mathrm{i}}^{\prime \prime \prime}=-2 \mathrm{~L}^{2} \cos \xi\left(\mathrm{a}_{13 \mathrm{i}} \cos ^{2} \beta_{3} \cos \xi+\mathrm{b}_{13 \mathrm{i}} \sin ^{2} \beta_{3} \cos \xi\right.$ $\left.+d_{13 i} \sin ^{2} \xi\right)$

$\mathrm{C}_{15 \mathrm{i}}^{\prime \prime \prime}=\mathrm{L}^{2} \cos ^{2} \xi\left(\mathrm{a}_{13 \mathrm{i}}^{2} \cos ^{2} \beta_{3}+\mathrm{b}_{13 \mathrm{i}}^{2} \sin ^{2} \beta_{3}+\mathrm{d}_{13 \mathrm{i}}^{2} \sin ^{2} \xi\right)-3 \mathrm{~h}^{2}$

$\mathrm{a}_{12 \mathrm{i}}=\left(\mathrm{a}_{12}-1\right)\left(\frac{\mathrm{g}}{\mathrm{L} \cos \xi}\right)+\left(\frac{1}{\mathrm{~L}}\right)\left(\mathrm{a}_{12} \mathrm{~d}_{1 \mathrm{i}}-\mathrm{d}_{2 \mathrm{i}}\right)$

$\mathrm{b}_{12 \mathrm{i}}=\left(\mathrm{b}_{12}-1\right)\left(\frac{\mathrm{g}}{\mathrm{L} \cos \xi}\right)+\left(\frac{1}{\mathrm{~L}}\right)\left(\mathrm{b}_{12} \mathrm{~d}_{1 \mathrm{i}}-\mathrm{d}_{2 \mathrm{i}}\right)$

$\mathrm{d}_{12 \mathrm{i}}=\left(\frac{1}{\mathrm{~L} \cos \xi}\right)\left(\mathrm{d}_{1 \mathrm{i}}-\mathrm{d}_{2 \mathrm{i}}\right)$

$\mathrm{a}_{23 \mathrm{i}}=\left(\mathrm{a}_{23}-1\right)\left(\frac{\mathrm{g}}{\mathrm{L} \cos \xi}\right)+\left(\frac{1}{\mathrm{~L}}\right)\left(\mathrm{a}_{23} \mathrm{~d}_{2 \mathrm{i}}-\mathrm{d}_{3 \mathrm{i}}\right)$

$b_{23 i}=\left(b_{23}-1\right)\left(\frac{g}{L \cos \xi}\right)+\left(\frac{1}{L}\right)\left(b_{23} d_{2 i}-d_{3 i}\right)$

$\mathrm{d}_{23 \mathrm{i}}=\left(\frac{1}{\mathrm{~L} \cos \xi}\right)\left(\mathrm{d}_{2 \mathrm{i}}-\mathrm{d}_{3 \mathrm{i}}\right)$ $\mathrm{a}_{13 \mathrm{i}}=\left(\mathrm{a}_{13}-1\right)\left(\frac{\mathrm{g}}{\mathrm{L} \cos \xi}\right)+\left(\frac{1}{\mathrm{~L}}\right)\left(\mathrm{a}_{13} \mathrm{~d}_{1 \mathrm{i}}-\mathrm{d}_{3 \mathrm{i}}\right)$

$b_{13 i}=\left(b_{13}-1\right)\left(\frac{g}{L \cos \xi}\right)+\left(\frac{1}{L}\right)\left(b_{13} d_{1 i}-d_{3 i}\right)$

$\mathrm{d}_{13 \mathrm{i}}=\left(\frac{1}{\mathrm{~L} \cos \xi}\right)\left(\mathrm{d}_{1 \mathrm{i}}-\mathrm{d}_{3 \mathrm{i}}\right)$

$\mathrm{a}_{12}=\frac{\cos \beta_{1}}{\cos \beta_{2}}, \mathrm{a}_{23}=\frac{\cos \beta_{2}}{\cos \beta_{3}}, \mathrm{a}_{13}=\frac{\cos \beta_{1}}{\cos \beta_{3}}$

$b_{12}=\frac{\sin \beta_{1}}{\sin \beta_{2}}, b_{23}=\frac{\sin \beta_{2}}{\sin \beta_{3}}, b_{13}=\frac{\sin \beta_{1}}{\sin \beta_{3}}$

\section{References}

[1] Gough, V.E., and Whitehall, S.G, "Universal Tyre Test Machine", Proc. Of the $9^{\text {th }}$ International congress of F.I.S.T.A., 117, ( 1962), pp. 117-135

[2] Stewart, D., “ A platform with six Degrees of Freedom", Proc. Inst. Mech. Engg., 180(15) , (1965), pp. 371-386

[3] Yangmini Li, QingsongXu R.F, “ Kinematics and dexterity analysis for a novel 3-dof Translational parallel manipulator", J of IEEE 07803-8914-X/05, (2005), pp. 2944-2949.

[4] Yangmin Li, qingsongXu R.F, "Kinematic Analysis and design of a new 3-dof translational parallel manipulator". J of ASME Vol. 128, (2006), PP. 729-737

[5] Meng-Shiun Tsai, Ting-NungShiau, Yi-Jeng Tsai and Tsann-Huei Chang R.F, "Direct Kinematic analysis of a 3-PRS parallel mechanism", J of Mechanism and Machine Theory, Vol.38, (2003), pp.71-83

[6] J.A.Carretero, R.P Podhorodeski, M.A.Nahon R.F, "Kinematic analysis and optimization of a new three degree-of-freedom Spatial parallel manipulator", J of Mechanical Design Vol.122, (2000), pp. 17-24

[7] K. Lee ,D. K. Shah, "Kinematic analysis of a three degrees of freedom in- parallel actuated manipulator", in: Proceedings of the IEEE International Conference on Robotics and Automation, Vol. 1, (1987), pp. 345-350

[8] Yangmin Li, QingsongXu R.F, "Kinematic analysis of a 3-PRS parallel manipulator", $J$ of Robotics and Computer-Integrated Manufacturing Vol.23, (2007), pp.395-408

[9] Xin-Jun Liu and Jongwon Kim R.F," A New Three-Degree-ofFreedom parallel Manipulator", J of IEEE , May (2002), pp.11551160

[10] FarhadTahmasebi R.F, " Kinematics of a new high-precision threedegree- of- freedom parallel manipulator", J of ASME, Vol. 129, (2007), pp. $320-325$ 